\title{
EL ANÁLISIS BAYESIANO Y LA PRECISIÓN DE LOS VALORES DE LA HEREDABILIDAD EN ESPECIES PERENNES
}

\author{
THE BAYESIAN APPROACH AND THE PRECISION OF THE HERITABILITY ESTIMATE IN \\ PERENNIAL SPECIES
}

\author{
Freddy Mora ${ }^{1}$ Carlos Alberto Scapim ${ }^{2}$ Ronald José Barth Pinto ${ }^{3}$ \\ RESUMEN
}

Los objetivos del presente trabajo fueron evaluar la precisión del valor estimado de la heredabilidad determinada por medio de la desviación estándar, considerando un enfoque Bayesiano, y comparar tal estimativa con el procedimiento clásico. Se utilizaron datos de un ensayo de progenie con 39 familias de Eucalyptus cladocalyx. La variable dependiente usada fue el diámetro basal del fuste medido a los seis años de edad. El método Bayesiano fue implementado por medio del algoritmo de Cadenas Independientes, con informaciones a priori informativas, el cual entregó bajos valores de desviaciones estándar de la heredabilidad, si comparado con la estimación clásica de Robertson y distribución a priori de Jeffreys (la cual es una clase de distribución a priori no informativa y a menudo impropia). El método de análisis Bayesiano es una herramienta de inferencia valiosa para la evaluación genética de especies perennes, ya que considera la variabilidad de los parámetros por medio de las distribuciones a posteriori.

Palabras-clave: inferencia Bayesiana; algoritmo de cadenas independientes; Eucalyptus; heredabilidad.

\section{ABSTRACT}

The objectives of the present work were to evaluate the precision of the estimate of heritability, which was determined by standard error, considering a Bayesian approach, and to compare such estimate with the classic procedure. Data from progeny trial of 39 Eucalyptus cladocalyx families were used. Trunk basal diameter (measured at age 6) was the dependent variable analyzed. The Bayesian approach was implemented by using the Independence Chain algorithm with informative priors, which had a lower standard deviation of the heritability than both the classic method of Robertson and Jeffreys' prior distribution (Jeffreys' prior is in fact a class of uninformative and often improper prior distributions). The Bayesian approach is a valuable inference tool for the genetic evaluation of perennial species, because it considers the variability of the parameters by using posterior distributions.

Keywords: Bayesian inference; independence chain algorithm; Eucalyptus; heritability.

\section{INTRODUCCIÓN}

En el contexto de la metodología de los modelos lineales mixtos, la predicción de los efectos aleatorios del modelo es realizada utilizando el método de la Mejor Predicción Lineal Insesgada (BLUP; Best Linear Unbiased Prediction), cuyo procedimiento es realizado maximizando la función de densidad conjunta, que considera tanto los efectos fijos como los aleatorios del modelo. A su vez, la Mejor Estimación Linear Insesgada (BLUE; Best Linear Unbiased Estimation) de los efectos fijos puede ser obtenida por medio del sistema de ecuaciones de modelos mixtos (RESENDE, 2002; ARNHOLD et al., 2009).

La estimación precisa de los componentes de varianza es de fundamental importancia en los programas de mejoramiento genético, ya que éstos permiten predecir el valor genético de los genotipos y la identificación de individuos genéticamente superiores. Estos estimadores paramétricos pueden variar como consecuencia de las diferencias genéticas de la población, del ambiente, del tipo de análisis y del método de estimación de los componentes de (co) varianza, entre otros (DE PAULA, 2006). En este sentido, la predicción de los efectos aleatorios del modelo mixto, por ejemplo: la predicción de los valores genéticos de las plantas, depende de las estimaciones de los componentes de varianza, los cuales han sido estimados frecuentemente usando el procedimiento de las esperanzas de cuadrados medios (o tipo I de Henderson). Sin

1. Ingeniero Forestal, Dr., Professor de la Facultad de Ciencias Forestales, Universidad de Concepción, Victoria 631, Barrio Universitario, Concepción, Chile.morapoblete@gmail.com, freddymora@udec.cl

2. Ingeniero Agronomo, Dr., Professor Associado de la Universidade Estadual de Maringá, Departamento de Agronomia, Bloco J45, Avenida Colombo 5790, CEP: 87020-900, Maringá (PR). cascapim@uem.br

3. Ingeniero Agronomo, PhD., Professor Adjunto, Universidade Estadual de Maringá, Departamento de Agronomia, Bloco J45, Avenida Colombo 5790, CEP: 87020-900, Maringá (PR).rjbpinto@uem.br

Recebido para publicação em 12/11/2008 e aceito em 22/09/2009. 
embargo, cuando existe perdidas de información en el conjunto de datos agronómicos ha sido comúnmente preferido el método de la Máxima Verosimilitud Restringida (REML; Restricted Maximum Likelihood) (RESENDE, 2002).

Por otra parte, el procedimiento Bayesiano, usando diferentes métodos de Monte-Carlo y Cadenas de Markov (MCMC) tal como o algoritmo de Gibbs, es indicado como una alternativa al método de estimación / predicción REML/BLUP (CAPPA y CANTET, 2006; GONÇALVES-VIDIGAL et al., 2008; MORA et al., 2008a; 2006; MORA et al., 2009; RESENDE, 2002). En el mejoramiento vegetal, diversos estudios confirmaron una fuerte asociación entre el enfoque Bayesiano y el procedimiento tradicional de estimación / predicción, REML/BLUP. Blasco (2001) ratificó que BLUP puede ser considerado un estimador Bayesiano, el cual es construido usando una distribución a priori uniforme (flat) para los efectos ambientales, y una distribución a priori normal para los efectos genéticos.

Gianola y Fernando (1986) propusieron usar el enfoque Bayesiano como método general de inferencia para resolver un gran número de problemas en el mejoramiento, lineales o no lineales, especialmente en situaciones donde existe incertidumbre sobre todos los parámetros de posición y de dispersión. Las primeras aplicaciones de este paradigma se basaron en las aproximaciones Gaussianas a la distribución posterior conjunta o a las distribuciones posteriores marginalizadas parcialmente, debido a las dificultades técnicas que fueron encontradas.

Del punto de vista del análisis clásico, Robertson (1959) sugirió que la varianza del valor estimado de la heredabilidad calculada en base a la covarianza de medios hermanos equivale aproximadamente a:

$$
\operatorname{Var}\left(\mathrm{h}^{2}\right)=32 \mathrm{~h}^{2} / \mathrm{N} \mathrm{p}
$$

Siendo $N$ el número de individuos por familias de medios-hermanos y $p$ el número de familias de medios-hermanos. Por lo tanto, es posible determinar el número total de individuos escogiendo a priori un valor deseado de desviación estándar para el valor estimado de la heredabilidad.

Resende et al. (1998) mencionaron que la obtención de estimadores confiables de los parámetros genéticos es requisito necesario para una adecuada predicción de valores genéticos. En su estudio, Resende et al. (1998) afirmaron que la desviación estándar de la heredabilidad no debería ser superior a $20 \%$ del valor estimado del parámetro para obtener informaciones precisas del parámetro genético e de los valores genéticos. Por ejemplo, para un valor de heredabilidad (sensu estricto) de $\mathrm{h}^{2}=0,35$, el valor estimado de la desviación estándar sería 0,07 .

Los objetivos analíticos del presente trabajo fueron examinar la precisión del valor estimado de la heredabilidad determinada por medio de la desviación estándar, considerando un enfoque Bayesiano, y comparar tal estimativa con el procedimiento clásico.

\section{MATERIALES Y MÉTODOS}

Se utilizó un conjunto de datos provenientes de ensayos de progenie de Eucalyptus cladocalyx pertenecientes al Instituto de Investigación Forestal, del Ministerio de Agricultura de Chile (MORA, 2006; MORA et al., 2007; MORA et al., 2009). La característica cuantitativa utilizada fue el diámetro basal del fuste medido en arboles de seis años de edad.

El diseño experimental fue de bloques completos al azar, con 39 familias, como los tratamientos principales, y 30 bloques en esquema single-tree plot. La densidad de plantación fue de, aproximadamente, 1667 árboles ha ${ }^{-1}$; equivalentes a un espaciamiento de $2 \times 3 \mathrm{~m}$.

Los componentes de varianza, heredabilidad y la predicción de valores genéticos obtenidos por el método Bayesiano se basaron el valor promedio, mediana y moda de cada muestra a posteriori de tamaño 50.000, generada por el algoritmo de Cadenas Independientes, una variante de los métodos de Monte Carlo Cadenas de Markov (MCMC: Markov Chain Monte Carlo). Se eliminaron las primeras 5.000 muestras de cada cadena.

Para el análisis Bayesiano se utilizó el procedimiento PROC MIXED (SAS-INSTITUTE, 1996) con la opción PRIOR. Se consideraron dos procedimientos teóricos:

1) Considerando distribuciones a priori no informativas para los componentes de varianza, o de Jeffreys, el cual es una clase de distribución a priori no informativa y a menudo impropia; procedimiento denominado aquí como BAYES1: 
PROC MIXED DATA=EUCA;

CLASS FAMILY BLOCK;

MODEL D = BLOCK;

RANDOM FAMILY / S;

PRIOR JEFFREYS / SEED=1978 OUT=BAYES1 ALG= INDCHAIN NSAMPLE=50000;

RUN;

2) Se construyeron un conjunto de datos con las informaciones a priori de los componentes de varianza (utilizando la densidad base), método denominado BAYES2:

DATA PRIOR;

INPUT DENSITY TYPE \$ PARM1 PARM2;

CARDS;

$1 \quad$ IG $\quad 18.336 \quad 16251$

2 IG $\quad 508.02 \quad 124516$

;

PROC MIXED DATA=EUCA;

CLASS FAMILY BLOCK;

MODEL D = BLOCK;

RANDOM FAMILY / S;

PRIOR DATA=PRIOR $/$ SEED $=1243$ OUT=BAYES2 ALG= INDCHAIN NSAMPLE=50000;

RUN;

En ambos casos las muestras a posteriori se analizaron con el procedimiento PROC UNIVARIATE (SAS-INSTITUTE, 1996) y SAS-INSIGHT:

PROC UNIVARIATE DATA=BAYES1 (BAYES2); WHERE SAMPLE > 5000;

VAR COVP1 COVP2 GAM1... GAM39;

RUN;

El modelo general asociado al experimento fue el siguiente:

$y=X \beta+Z \gamma+\varepsilon$

Donde $y$ es el vector de las respuestas observadas, $X$ es la matriz de delineamiento que corresponde al vector de parámetros del efecto del bloque $(\beta)$; $Z$ es la matriz de incidencia que corresponde al vector de parámetros del efecto genotípico $(\gamma)$; y $\varepsilon$ es el vector de residuos. Se asume que $\gamma$ y $\varepsilon$ son independientes y con distribución normal univariada, con promedio 0 y matriz de varianza $\mathrm{G}$ y $\mathrm{R}$, respectivamente.

Definimos $\theta$ como el vector de componentes de varianza. La función de densidad conjunta de $(\beta, \gamma, \theta)$ es dado por:

$$
f(\beta, \gamma, \theta \mid y)=f(\beta, \gamma \mid \theta, y) f(\theta \mid y)
$$

La especificación de cada distribución a priori de los elementos del modelo es dado por (RODOVALHO et al., 2008; MORA et al., 2007):

$$
\begin{aligned}
& f\left(y \mid \beta, \gamma, \sigma_{e}^{2}, \sigma_{f}^{2}\right) \sim N(X \beta+Z \gamma, R) \\
& f(\beta) \propto 1 \\
& f\left(\gamma \mid \sigma_{f}^{2}\right) \sim N(0, G) \\
& f\left(\sigma_{f}^{2}\right) \sim I G(a, b) \\
& f\left(\sigma_{e}^{2}\right) \sim I G(c, d)
\end{aligned}
$$

Los componentes de varianzas tienen distribución a priori Gama Inversa $(\sim \mathrm{IG})$, en el caso de BAYES2, y Jeffreys para el caso de BAYES1. La función de densidad a priori del parámetro de efecto de Bloque es asumida como uniforme. 
La distribución a posteriori conjunta es definida como:

$$
f\left(\beta, \gamma, \sigma_{e}^{2}, \sigma_{f}^{2} \mid y\right) \propto f(\beta) f\left(\gamma \mid \sigma_{f}^{2}\right) f\left(\sigma_{f}^{2}\right) f\left(\sigma_{e}^{2}\right) f\left(y \mid \beta, \gamma, \sigma_{e}^{2}, \sigma_{f}^{2}\right)
$$

\section{RESULTADOS Y DISCUSIÓN}

En la Tabla 1 se presentan las estimaciones de los componentes de varianza y de la heredabilidad $\left(h^{2}\right)$ para la característica diámetro basal, medida en árboles de 39 familias de E. cladocalyx, usando un enfoque Bayesiano. Ambos procedimientos, estimaron similares valores de heredabilidad, pero existieron diferencias en las estimaciones de la desviación estándar de este parámetro.

TABLA 1: Estimaciones de los componentes de varianza familiar $\left(\sigma_{f}^{2}\right)$, residual $\left(\sigma_{e}^{2}\right)$ y heredabilidad $\left(h^{2}\right)$ para el diámetro del fuste, calculados en 39 familias de Eucalyptus cladocalyx, usando el método Bayesiano.

TABLE 1: Estimates of variance components: family $\left(\sigma_{f}^{2}\right)$, residual $\left(\sigma_{e}^{2}\right)$, and heritability $\left(h^{2}\right)$ for trunk basal diameter, which were calculated in 39 families of Eucalyptus cladocalyx using Bayesian method.

\begin{tabular}{|c|c|c|c|c|c|c|c|}
\hline \multirow[b]{2}{*}{ Parámetro } & \multicolumn{3}{|c|}{ BAYES1 } & \multicolumn{4}{|c|}{ BAYES2 } \\
\hline & $\sigma_{f}^{2}$ & $\sigma_{e}^{2}$ & $h^{2}$ & $\sigma_{f}^{2}$ & $\sigma_{e}^{2}$ & & $h^{2}$ \\
\hline Promedio & 24,75 & 245,62 & & 0,364 & 23,83 & 245,29 & 0,353 \\
\hline Mediana & 23,47 & $245,3($ & & 0,349 & 23,24 & 245,13 & 0,346 \\
\hline Moda & 21,54 & 244,92 & & 0,328 & 21,84 & 245,21 & 0,329 \\
\hline$\left[\operatorname{Var}\left(\sigma^{2}\right)\right]^{1 / 2}$ & 8,29 & 10,9 & & & 5,56 & 7,67 & \\
\hline$\left[\operatorname{Var}\left(\mathrm{h}^{2}\right)\right]^{1 / 2}$ & & & & 0,110 & & & 0,076 \\
\hline \multicolumn{8}{|c|}{ Intervalo de credibilidad } \\
\hline $95 \%$ & 40,11 & 264,15 & & 0,566 & 33,83 & 258,07 & 0,453 \\
\hline $5 \%$ & 13,69 & 228,19 & & 0,209 & 15,84 & 233,01 & 0,241 \\
\hline
\end{tabular}

BAYES1: distribuciones a priori no informativas (Jeffreys) para los componentes de varianza. BAYES2: distribuciones a priori con algún grado de información.

Del punto de vista clásico, de acuerdo con la fórmula de Robertson (1959), la desviación estándar de la heredabilidad (considerando un valor de $h^{2}=0,35$ ) en el presente estudio es:

$$
\sqrt{\operatorname{Var}\left(\mathrm{h}^{2}\right)}=\sqrt{32 \mathrm{~h}^{2} / \mathrm{Np}}=\sqrt{32 \cdot 0,35 / 30 \cdot 39}=0,0978
$$

O sea, aproximadamente $28 \%$ del valor estimado para la heredabilidad. Para obtener un valor estimado de la heredabilidad de $20 \%$ (RESENDE et al., 1998) de la desviación estándar $\left(\left[\operatorname{Var}\left(\mathrm{h}^{2}\right)\right]^{1 / 2}=0,07\right)$ serían necesarias 76 familias de medios-hermanos.

Con el procedimiento Bayesiano, utilizando informaciones a priori informativas de los componentes de varianza (BAYES2), fue posible obtener un valor razonable de la desviación estándar $\left(\left[\operatorname{Var}\left(\mathrm{h}^{2}\right)\right]^{1 / 2}=0.076\right)$; un valor muy cercano al valor clásico, pero considerando sólo 39 progenies.

Otra ventaja de los procedimientos Bayesianos es la posibilidad de usar los limites de confianza (o regiones de credibilidad en el contexto Bayesiano) como una alternativa de estimación de los parámetros genéticos, incluyendo los valores de predicción de los efectos genotípicos, los cuales son obtenidos directamente de la distribución a posteriori (WRIGHT et al., 2000; NOGUEIRA et al., 2003). En el presente estudio, si el genetista desea trabajar utilizando estimaciones por intervalo en el contexto Bayesiano, la familia Fam22 (Tabla 2) podría no ser seleccionada ya que dentro de la región de los limites de credibilidad se incluyen valores negativos de predicción, indicando que la predicción del efecto familiar pueden ser inferiores al promedio de la población (línea base). Consecuentemente, las seis primeras familias serían seleccionadas (equivalentes al 15,4\%) en función del valor genético del diámetro, estimado por medio de la utilización de las regiones de credibilidad. Este argumento está en concordancia con el estudio de Mora y Perret (2007) y Mora et al. (2008b). 
TABLA 2: Estimaciones de los valores promedios a posteriori de la predicción del efecto familiar de las siete mejore familias de Eucalyptus.

TABLE 2: Estimates of posterior mean values of the prediction of family effect for the seven best families of Eucalyptus.

\begin{tabular}{|c|c|c|c|c|c|c|c|c|}
\hline \multirow{3}{*}{ Familia } & \multicolumn{4}{|c|}{ BAYES1 } & \multicolumn{4}{|c|}{ BAYES2 } \\
\hline & \multirow{2}{*}{$\begin{array}{l}\text { Predicción } \\
\text { (P) }\end{array}$} & \multirow{2}{*}[\operatorname{Var}(\mathrm{P})]{$^{1 / 2}$} & \multicolumn{2}{|c|}{ IC (90\%) } & \multirow{2}{*}{$\begin{array}{l}\text { Predicción } \\
\text { (P) }\end{array}$} & \multirow{2}{*}[\operatorname{Var}(\mathrm{P})]{$^{1 / 2}$} & \multicolumn{2}{|c|}{ IC (90\%) } \\
\hline & & & Inferior & Superior & & & Inferior & Superior \\
\hline Fam30 & 8,10 & 2,68 & 3,75 & 12,54 & 8,11 & 2,62 & 3,83 & 12,49 \\
\hline Fam14 & 6,42 & 2,64 & 2,12 & 10,81 & 6,43 & 2,60 & 2,16 & 10,75 \\
\hline Fam26 & 6,05 & 2,59 & 1,84 & 10,36 & 6,07 & 2,57 & 1,85 & 10,30 \\
\hline Fam7 & 5,37 & 2,60 & 1,17 & 9,69 & 5,37 & 2,58 & 1,16 & 9,66 \\
\hline Fam28 & 4,68 & 2,57 & 0,47 & 8,90 & 4,64 & 2,56 & 0,42 & 8,86 \\
\hline Fam29 & 4,60 & 2,59 & 0,39 & 8,89 & 4,59 & 2,56 & 0,37 & 8,79 \\
\hline Fam22 & 3,76 & 2,67 & $-0,59$ & 8,19 & 3,76 & 2,66 & $-0,58$ & 8,15 \\
\hline
\end{tabular}

BAYES1: distribuciones a priori no informativas (Jeffreys) para los componentes de varianza. BAYES2: distribuciones a priori con algún grado de información. IC: intervalo de credibilidad. [Var $(\mathrm{P})]^{1 / 2}$ : desviación estándar del valor genético.

Ambos procedimientos Bayesianos (BAYES1 y BAYES2) entregaron resultados idénticos para la selección de familias de Eucalyptus, evidenciando robustez en las estimativas a posteriori de los valores de predicción genética. $\mathrm{O}$ sea, el resultado de predicción fue estable e independiente de la información de la distribución a priori.

Intervalos de confianza en el contexto clásico pueden eventualmente ser construidos por medio de técnicas semi-paramétricas de Bootstrap, como definido por Efron (1979), sin embargo, en el caso de la Mejor Predicción Linear Insesgada (BLUP), Morris (2002) aclara que algunas de las propiedades deseadas de este procedimiento podrían ser pérdidas (MORA y PERRET, 2007). La conclusión del trabajo de Morris se resume en que BLUP ya no es "mejor predicción" si viene de un proceso de Bootstrap.

Las ventajas confirmadas en este estudio, están en concordancia con Resende (2002), quien mencionó las siguientes conclusiones en relación a la inferencia Bayesiana:

1)El análisis Bayesiano propicia resultados adicionales a los obtenidos por el método clásico, destacándose los intervalos de confianza Bayesianos para las estimaciones de parámetros genéticos, valores genéticos y ganancia genética;

2)Las estimaciones de los parámetros genéticos obtenidas del algoritmo de Gibbs pueden ser más precisas que las estimaciones hechas por el método REML/BLUP;

3)El análisis Bayesiano es una técnica elegante y flexible que permite la estimación simultánea de los parámetros genéticos, efectos "fijos" y los valores genéticos de manera precisa, incluyendo muestras de tamaño finito. Sin embargo, el autor afirma que para la obtención de una precisión adecuada en el método Bayesiano es recomendable que el investigador tenga una cuantidad suficiente de información experimental.

Además del algoritmo de Gibbs, otros algoritmos derivados de los métodos MCMC son utilizados en conjunto con la teoría de modelos lineares mistos. Algunos ejemplos son: Cadenas Independientes (IC: Independence Chain; utilizado en este estudio) y Muestreo por Importancia (IS: Importance Sampling) ambos disponibles en SAS (SAS-INSTITUTE, 1996; WOLFINGER y KASS, 2000). Estos algoritmos son usados para la construcción de intervalos de credibilidad Bayesianos de los componentes de varianza (WOLFINGER y KASS, 2000), los cuales son calculados directamente a partir de la distribución $a$ posteriori.

Los resultados del presente estudio están en concordancia con Wolfinger y Kass (2000), quienes examinaron regiones de credibilidad para funciones generales de componentes de varianza usando la variante del método de MCMC, el algoritmo IC. Aquí, la distribución base para los efectos aleatorios es considerada exacta y, por lo tanto, las muestras MCMC son rechazadas solamente si éstas no están en el espacio del parámetro, el cual puede ocurrir, por ejemplo, si un componente de varianza individual es estimado con valor negativo. De este modo, el algoritmo IC es un caso especial de muestreo de rechazo (TIERNEY, 1994). 


\section{CONCLUSIONES}

El método Bayesiano permitió la obtención de bajos valores de desviaciones estándar de la heredabilidad, los cuales fueron inferiores a la estimación clásica para familias de medios-hermanos.

Se confirmó que la metodología Bayesiana es considerada una herramienta de inferencia importante en la evaluación genética de especies perennes, ya que lleva en consideración la variabilidad real existente en todos los parámetros de interés.

\section{AGRADECIMIENTOS}

Cordiales agradecimientos a Sandra Perret, Gerente Regional del Instituto de Investigación Forestal (INFOR) del Ministerio de Agricultura de Chile, quién gentilmente proveyó los datos para la realización de esta investigación.

\section{REFERENCIAS BIBLIOGRÁFICAS}

ARNHOLD, E. et al. Evaluation of top-cross popcorn hybrids using mixed linear model methodology. Chilean Journal of Agriculture Research, Santiago, v. 69, n. 1, p. 46-53, jan./mar. 2009.

BLASCO, A. The Bayesian controversy in animal breeding. Journal of Animal Science, Champaign, v. 79, n. 8, p. 2023-2046, ago. 2001.

CAPPA, E. P.; CANTET, R. J. C. Bayesian inference for normal multiple-trait individual-tree models with missing records via full conjugate Gibbs. Canadian Journal of Forest Research, Ottawa, v. 36, n. 5, p. 1276-1285, mai. 2006. DE PAULA, M. C. Heterogeneidade de variâncias e interação genótipo $\mathbf{x}$ ambiente para produção de leite em bovinos da raça holandesa no estado do Paraná. Maringá: UEM, 2006. 84 f. Tese (Doutorado em Zootecnia) Universidade Estadual de Maringá, 2006.

EFRON, B. Bootstrap methods: another look at the jackknife. The Annals of Statistics, Beachwood, v. 7, n. 1, p. 1-26, jan. 1979.

GIANOLA, D.; FERNANDO, R. L. Bayesian methods in animal breeding theory. Journal of Animal Science, Champaign, v. 63, n. 1, p. 217-277, set. 1986.

GONÇALVES-VIDIGAL, M. C. et al. Heritability of quantitative traits in segregating common bean families using a Bayesian approach. Euphytica, Wageningen, v. 164, n. 2, p. 551-560, nov. 2008.

MORA, F. Heredabilidad y valor genético (REML/BLUP) en genotipos de un eucalipto tolerante a la sequía, en el norte de Chile. Ciência Florestal, Santa Maria, v. 16, n. 2, p. 145-151, abr./jun. 2006.

MORA, F.; PERRET, S. Aplicación de técnicas bayesianas en el análisis genético de árboles forestales. Bosque, Valdivia, v. 28, n. 3, p. 198-206, set./dez. 2007.

MORA, F. et al. Source-dependent blooming variability of Eucalyptus cladocalyx in the Region of Coquimbo, Chile. Ciencia e Investigación Agraria, Santiago, v. 34, n. 2, p. 99-106, mai./ago. 2007.

MORA, F. et al. Bayesian analysis of the genetic control of survival in F3 families of common bean. Chilean Journal of Agriculture Research, Chillán, v. 68, n. 4, p. 334-341, dez. 2008a.

MORA, F. et al. Genetic evaluation of olive clones in the Atacama desert, Chile. Agrociencia, Montecillo, v. 42, n. 7 , p. 777-786, out./nov. 2008b.

MORA, F. et al. Genetic variation for early flowering, survival and growth in sugar gum (Eucalyptus cladocalyx F. Muell) in southern Atacama Desert. Euphytica, Wageningen. Disponível em: (http://www.springerlink.com/content/d78x173425v37338/fulltext.html) > Acesso em: 08 de julho de 2009.

MORRIS, J. S. The BLUPs are not "best" when it comes to bootstrapping. Statistics and Probability Letters, Madison, v. 56, n. 4, p. 425-430, fev. 2002.

NOGUEIRA, D. A. et al. Análises clássica e Bayesiana de um modelo misto aplicado ao melhoramento animal: uma ilustração. Ciência e Agrotecnologia, Lavras, v. 27, n. especial, p. 1614-1624, dez. 2003.

RESENDE, M. D. V. Genética biométrica e estatística no melhoramento de plantas perenes. Brasília, Brasil: Embrapa Informação Tecnológica, 2002. 975 p.

RESENDE, M. D. V. et al. Efeito do tamanho amostral na estimativa da herdabilidade em espécies perenes. Revista Floresta, Curitiba, v. 28, n. 1-2, p. 51-63, jun./dez. 1998.

ROBERTSON, A. Experimental design in the evaluation of genetic parameters. Biometrics, Raleigh, v. 15, n. 2, p. 219-226, jun. 1959.

RODOVALHO, M. A. et al. Survival heritability in 169 families of white grain popcorn: a Bayesian approach. Ciencia e Investigación Agraria, Santiago, v. 35, n. 3, p. 255-260, set./dez. 2008. 
SAS-INSTITUTE. Statistical analysis system. User's guide. Cary, USA: SAS Institute, 1996. 956 p.

TIERNEY, L. Markov chains for exploring posterior distributions (with discussion). Annals of Statistics, v. 23, n. 4, p. 1701-1762, dez. 1994.

WOLFINGER, R. D.; KASS, R. E. Non-conjugate Bayesian analysis of variance component models. Biometrics, Beachwood, v. 56, n. 3, p. 768-774, set. 2000.

WRIGHT, D. R.; STERN, H. S.; BERGER, P. J. Comparing traditional and Bayesian analyses of selection experiments in animal breeding. Journal of Agricultural, Biological, and Environmental Statistics, Alexandria, v. 5, n. 2, p. 240256, jun. 2000. 